hep-th/9806243

\title{
Lorentz covariance, higher-spin superspaces and self-duality
}

\author{
Chandrashekar Devchand ${ }^{a}$ and Jean Nuyts ${ }^{b}$ \\ devchand@mis.mpg.de, nuyts@umh.ac.be \\ ${ }^{a}$ Max-Planck-Institut für Mathematik in den Naturwissenschaften \\ Inselstraße 22-26, 04103 Leipzig, Germany \\ ${ }^{b}$ Physique Théorique et Mathématique, Université de Mons-Hainaut \\ 20 Place du Parc, 7000 Mons, Belgium
}

\begin{abstract}
Lorentz covariant generalisations of the notions of supersymmetry, superspace and selfduality are discussed. The essential idea is to extend standard constructions by allowing tangent vectors and coordinates which transform according to more general Lorentz representations than solely the spinorial and vectorial ones of standard lore. Such superspaces provide model configuration spaces for theories of arbitrary spin fields. Our framework is an elegant one for handling higher-dimensional theories in a manifestly $\mathrm{SO}(3,1)$ covariant fashion. A further application is the construction of a hierarchy of solvable Lorentz covariant systems generalising four-dimensional self-duality.
\end{abstract}

\section{Introduction}

There exist various forms of higher dimensional fundamental building blocks for the observed four dimensional 'real world'. An important question is the recovery of four-dimensional Lorentz invariance, the most important observed symmetry in physics. It often seems desirable to have a framework in which the higher dimensional objects transform covariantly under $S O(3,1)$ and therefore have some intrinsic four-dimensional nature. Recent developments in string physics, moreover, seem to require an extension of the standard notions of supersymmetry and superspace. Over the past couple of years, we have been investigating $S O(4, \mathbb{C})$-covariant superalgebras which provide generalisations of supersymmetry and a manifestly Lorentz-covariant framework for the investigation of spaces of dimensions greater than four.

Standard superspace is a homogeneous space constructed as the quotient of the super Poincaré group by the Lorentz group, with four bosonic coordinates $\left\{Y^{\alpha \dot{\alpha}}\right\}$ and four fermionic coordinates $\left\{Y^{\alpha}, Y^{\dot{\alpha}}\right\}$, transforming according to the vectorial $\left(\frac{1}{2}, \frac{1}{2}\right)$ and spinorial $\left(0, \frac{1}{2}\right)$ and $\left(\frac{1}{2}, 0\right)$ representations of the Lorentz group. Superspace can therefore be considered as a direct sum of $4 \mathrm{~d}$ Minkowski space with two $2 \mathrm{~d}$ spinorial spaces. Now, once the restriction to coordinates transforming solely according to the vectorial $\left(\frac{1}{2}, \frac{1}{2}\right)$ representation has been abandoned, one can ask whether one can build more general superspaces from direct sums of more general sets of representation spaces than the usual spinorial and vectorial ones; namely, spaces with coordinates from the set of general Lorentz tensors $\left\{Y^{\alpha_{1} \ldots \alpha_{2 s} \dot{\alpha}_{1} \ldots \dot{\alpha}_{2 \dot{s}}}\right\}$, symmetrical in the $\alpha$ indices

\footnotetext{
${ }^{1}$ Presented by Jean Nuyts at the International Conference on Particles, Fields and Gravitation, Eodz, Poland (April 15-19 1998); to be published in the Proceedings.
} 
and in the $\dot{\alpha}$ indices, for some collection of representations $\Lambda_{p}=\{(s, \dot{s})\}$, where $p$ denotes the maximum value of $s+\dot{s}$ occurring in the chosen set of representations.

This is clearly a way of parametrising a higher-dimensional space in a manifestly $4 \mathrm{~d}$ Lorentz covariant fashion. Usually, in higher-dimensional theories, the recovery of $4 \mathrm{~d}$ Lorentz covariance is achieved by having the extra coordinates transform as a bunch of Lorentz scalars. The idea here is to start with a set of Lorentz representations and hence fix the $4 \mathrm{~d}$ Lorentz structure to the coordinate system. For instance a simple bosonic extension of $4 \mathrm{~d}$ space with coordinates $\left\{Y^{\alpha \dot{\alpha}}, Y^{\alpha \beta \gamma \dot{\alpha}}\right\}$ has dimension $4+8=12$. For the sake of generality, we consider a graded vector space with the coordinates $\{Y(s, \dot{s})\}$ spanning a supercommutative $\mathbb{Z}_{2}$-graded algebra, $\mathcal{V}=\mathcal{V}_{0}+\mathcal{V}_{1} . \mathcal{V}_{0}$ (resp. $\mathcal{V}_{1}$ ) contains bosonic (resp. fermionic) coordinates if $2(s+\dot{s})$ is even (resp. odd). Each representation $Y(s, \dot{s})$ included, increases the bosonic (resp. fermionic) dimension of the hyperspace $\mathcal{M}$ by $(2 s+1)(2 \dot{s}+1)$.

\section{Spin-p Heisenberg superalgebras}

To describe such hyperspaces as the homogeneous spaces of some algebra, the super-Poincaré algebra needs to be extended to some algebra including higher-spin generators. Such enhancement of customary supersymmetry, going beyond the Haag-Łopusanski-Sohnius barrier, has been extensively studied by Fradkin and Vasiliev [1]. These authors were motivated by physical considerations to realise such higher-spin algebras on $4 \mathrm{~d}$ de Sitter space fields. Consistency of the dynamics required the inclusion of all spins, yielding infinite dimensional algebras realised on infinite chains of fields having spins all the way up to infinity.

Our approach [2, 3, 4] has been more abstract and does not require infinite dimensionality of the algebra. We consider some basically finite set of higher-spin generators (in addition

to the spin 1 and spin $\frac{1}{2}$ super-Poincaré generators); and we interpret the higher-spin generators $X_{\alpha_{1} \ldots \alpha_{2 s} \dot{\alpha}_{1} \ldots \dot{\alpha}_{2 \dot{s}}}$ as 'momenta' in extra dimensions parametrised by higher-spin coordinates. We do not make any a priori field theoretical or dynamical requirements; and we realise our algebras in flat space. The grading, however, remains a $\mathbb{Z}_{2}$ one, with all integer-spin representations in an even-statistics (bosonic) subspace $\mathcal{A}_{0}$ and all half-integer-spin representations in an odd-statistics (fermionic) subspace $\mathcal{A}_{1}$. Thus the Heisenberg superalgebra associated to standard (complexified) superspace with $\Lambda_{1}=\left\{\left(\frac{1}{2}, \frac{1}{2}\right),\left(\frac{1}{2}, 0\right),\left(0, \frac{1}{2}\right)\right\}$ and non-zero canonical supercommutation relations,

$$
\begin{array}{lll}
\left\{X_{\alpha}, X_{\dot{\beta}}\right\}=X_{\alpha \dot{\beta}}, & {\left[X_{\alpha}, Y^{\beta \dot{\beta}}\right]=\delta_{\alpha}^{\beta} Y^{\dot{\beta}},} & {\left[X_{\dot{\alpha}}, Y^{\beta \dot{\beta}}\right]=\delta_{\dot{\alpha}}^{\dot{\beta}} Y^{\beta}} \\
{\left[X_{\alpha \dot{\alpha}}, Y^{\beta \dot{\beta}}\right]=\delta_{\alpha}^{\beta} \delta_{\dot{\alpha}}^{\dot{\beta}},} & \left\{X_{\alpha}, Y^{\beta}\right\}=\delta_{\alpha}^{\beta}, & \left\{X_{\dot{\alpha}}, Y^{\dot{\beta}}\right\}=\delta_{\dot{\alpha}}^{\dot{\beta}},
\end{array}
$$

is generalised to a spin $p$ Heisenberg superalgebra $\mathcal{G}=\mathcal{A}+\mathcal{V}$, based on a set of representations $\Lambda_{p}=\{(s, \dot{s})\}$ appearing in $\mathcal{A}+\mathcal{V}$.

For any specific $(s, \dot{s}) \in \Lambda_{p}$, we label the $(2 s+1)(2 \dot{s}+1)$ components of the coordinate tensor $Y(s, \dot{s})$ as $Y\left(s, s_{3} ; \dot{s}, \dot{s}_{3}\right)$, where $s_{3}$ (resp. $\left.\dot{s}_{3}\right)$ run from $-s$ to $s$ (resp. from $-\dot{s}$ to $\dot{s}$ ) in integer steps. These are in one-to-one correspondence with the components in standard 2-spinor index notation, $Y^{\alpha_{1} \ldots \alpha_{2 s} \dot{\alpha}_{1} \ldots \dot{\alpha}_{2 \dot{s}}}$. The span of coordinates $\left\{Y\left(s, s_{3} ; \dot{s}, \dot{s}_{3}\right)\right\}$, for all $(s, \dot{s})$ in the chosen set $\Lambda_{p}$, is defined to be a supercommutative basis of the vector space $\mathcal{V}$. The corresponding tangent 
space algebra $\mathcal{A}$ is spanned by components $\left\{X\left(s, s_{3} ; \dot{s}, \dot{s}_{3}\right)\right\}$ of vector fields $\left\{X(s, \dot{s}) ;(s, \dot{s}) \in \Lambda_{p}\right\}$ in one-to-one correspondence with the coordinate tensors. Denoting

$$
S=(s, \dot{s}), \quad \bar{S}=\left\{s, s_{3} ; \dot{s}, \dot{s}_{3}\right\}, \quad R=(r, \dot{r}), \quad \bar{R}=\left\{r, r_{3} ; \dot{r}, \dot{r}_{3}\right\}, \quad \text { etc. },
$$

and defining the sign of the graded bracket as

$$
S \bullet R=R \bullet S=(-1)^{4(s+\dot{s})(r+\dot{r})+1},
$$

the most general Lorentz covariant superalgebra $\mathcal{A}$ of vector fields takes the form

$$
\begin{aligned}
& {[X(\bar{S}), X(\bar{R})]_{S \bullet R}} \\
& =\sum_{(v, \dot{v}) \in \Gamma(S, R) \cap \Lambda_{p}} C\left(s, s_{3}, r, r_{3} ; v, s_{3}+r_{3}\right) C\left(\dot{s}, \dot{s}_{3}, \dot{r}, \dot{r}_{3} ; \dot{v}, \dot{s}_{3}+\dot{r}_{3}\right) \\
& \quad \times t(s, \dot{s}, r, \dot{r}, v, \dot{v}) X\left(v, s_{3}+r_{3} ; \dot{v}, \dot{s}_{3}+\dot{r}_{3}\right) .
\end{aligned}
$$

Here $C\left(s, s_{3}, r, r_{3} ; v, s_{3}+r_{3}\right)$ are $S U(2)$ Clebsch-Gordan coefficients and $\Gamma(S, R)$ denotes the indices in the double Clebsch-Gordon decomposition:

$$
\Gamma(S, R)=\{(v, \dot{v}) ; \quad v \in \gamma(s, r), \quad \dot{v} \in \gamma(\dot{s}, \dot{r})\} ; \quad \gamma(s, r)=\{s+r, s+r-1, \ldots,|s-r|\} .
$$

The $t(s, \dot{s}, r, \dot{r}, v, \dot{v})$ 's are structure constants. Subject to superskewsymmetry requirements inherited from the graded bracket and quadratic constraints implied by the super Jacobi identities, they parametrise the moduli space of superalgebras with relations (1).

The vector fields $X \in \mathcal{A}=\mathcal{A}_{0}+\mathcal{A}_{1}$ act as superderivations on functions of the $Y$ 's. We require that the action of $\mathcal{A}$ on $\mathcal{V}$ corresponds to a linear transformation. The combined vector space thus has supercommutation relations of a generalised Heisenberg superalgebra, with the most general Lorentz covariant relations between the $X$ 's and the $Y$ 's taking the form

$$
\begin{aligned}
& {[X(\bar{S}), Y(\bar{R})]_{S \bullet R}} \\
& =\sum_{(v, \dot{v}) \in \Gamma(S, R) \cap \Lambda_{p}} C\left(s, s_{3}, r, r_{3} ; v, s_{3}+r_{3}\right) C\left(\dot{s}, \dot{s}_{3}, \dot{r}, \dot{r}_{3} ; \dot{v}, \dot{s}_{3}+\dot{r}_{3}\right) \\
& \times u(s, \dot{s}, r, \dot{r}, v, \dot{v}) Y\left(v, s_{3}+r_{3} ; \dot{v}, \dot{s}_{3}+\dot{r}_{3}\right) \\
& +C\left(s, s_{3}, s,-s_{3} ; 0,0\right) C\left(\dot{s}, \dot{s}_{3}, \dot{s},-\dot{s}_{3} ; 0,0\right) c(s, \dot{s}) \delta_{s r} \delta_{\dot{s} \dot{r}} \delta_{s_{3}+r_{3}, 0} \delta_{\dot{s}_{3}+\dot{r}_{3}, 0}
\end{aligned}
$$

The $u$ 's are further structure constants and the 'central' parameters $c(s, \dot{s})$ determine a bilinear pairing between the $X$ 's and the $Y$ 's,

$$
<., .>: \mathcal{A} \times \mathcal{V} \rightarrow \mathcal{C}=\left\{c(s, \dot{s}) ;(s, \dot{s}) \in \Lambda_{p}\right\}
$$

given by

$$
<X(\bar{S}), Y(\bar{R})>=c(s, \dot{s}) C\left(s, s_{3}, s,-s_{3} ; 0,0\right) C\left(\dot{s}, \dot{s}_{3}, \dot{s},-\dot{s}_{3} ; 0,0\right) \delta_{s r} \delta_{\dot{s} \dot{r}} \delta_{s_{3}+r_{3}, 0} \delta_{\dot{s}_{3}+\dot{r}_{3}, 0}
$$

where the CG coefficients $C\left(s, s_{3}, s,-s_{3} ; 0,0\right)$ denote Wigner's 'metric' invariant in the representation space of fixed spin $s$. 
Therefore, requiring Lorentz covariance determines the space of a priori allowed structure constants $\{t, u, c\}$. The combined vector space $\mathcal{G}=\mathcal{A}+\mathcal{V}$ forms a superalgebra if these structure constants are subject to quadratic equations, the satisfaction of which make the super Jacobi identities amongst the $X$ 's and the $Y$ 's automatic. These quadratic equations define the moduli space of $\operatorname{spin} p$ Lorentz covariant Heisenberg superalgebras. For any fixed set $\Lambda_{p}$, these equations allow determination using explicit values for Clebsch-Gordan coefficients and $6 j$ symbols (from e.g. (4)). Particular solutions of these equations provide concrete examples of Lorentz covariant spin $p$ superspaces; and they exist for any finite or infinite $p$.

\section{Spin 2 superspaces}

The hyperspaces $\mathcal{M}$ serve as models for configuration spaces, or for moduli spaces of solutions, of Lorentz invariant field theories; and the supercommutations relations for $\mathcal{G}$ provide canonical supercommutation relations for the corresponding phase spaces. They therefore provide an algebraic description of the local symplectic structure. With this application in mind, it is clear that algebras including spins up to two are of possible significance for the canonical quantisation of gravity and supergravity theories. By including generators transforming according to every Lorentz representation (with unit multiplicity) having spin up to two, the complete set of quadratic equations for the Lorentz covariant structure constants were determined in «4. These defining equations for spin two Heisenberg superalgebras are highly overdetermined. Nevertheless, non-trivial solutions can indeed be found. Explicit examples of algebras $\mathcal{G}$ were presented

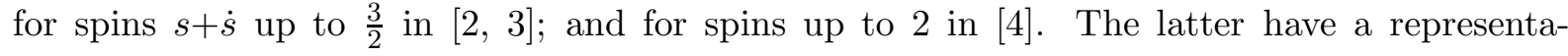
tion content reminiscent of simple (super)gravity theories: a metric represented by canonically conjugate variables $X, Y$ transforming as $(0,0) \oplus(1,1)$ coupled to single copies of other (spin $<2)$ representations, including Rarita-Schwinger representations $\left(1, \frac{1}{2}\right) \oplus\left(\frac{1}{2}, 1\right)$ corresponding to gravitino phase-space variables. Concrete physical application to the canonical quantisation of supergravity theories, however, remains for future investigation.

\section{Generalised self-duality}

A further application of these hyperspaces considers gauge fields on $\mathcal{M}$ [2, 3] : Associating a gauge potential $A$ (depending on the $Y$ 's) to each of these generalised derivatives, we define gauge-covariant derivatives $D(s, \dot{s})=X(s, \dot{s})+A(s, \dot{s})$. The curvature components $F\left(s, s_{3} ; \dot{s}, \dot{s}_{3}\right)$ which are a priori non-zero are in turn defined in a consistent fashion by:

$$
\begin{aligned}
& {[D(\bar{S}), D(\bar{R})]_{S \bullet R}} \\
& -\sum_{(v, \dot{v}) \in \widehat{\Gamma}(S, R) \cap \Lambda_{p}} C\left(s, s_{3}, r, r_{3} ; v, s_{3}+r_{3}\right) C\left(\dot{s}, \dot{s}_{3}, \dot{r}, \dot{r}_{3} ; \dot{v}, \dot{s}_{3}+\dot{r}_{3}\right) \\
& \quad \times t(s, \dot{s}, r, \dot{r}, v, \dot{v}) D\left(v, s_{3}+r_{3} ; \dot{v}, \dot{s}_{3}+\dot{r}_{3}\right) \\
& =\quad \sum_{(v, \dot{v}) \in \widehat{\Gamma}(S, R)} C\left(s, s_{3}, r, r_{3} ; v, s_{3}+r_{3}\right) C\left(\dot{s}, \dot{s}_{3}, \dot{r}, \dot{r}_{3} ; \dot{v}, \dot{s}_{3}+\dot{r}_{3}\right) \\
& \quad \times F\left(v, s_{3}+r_{3} ; \dot{v}, \dot{s}_{3}+\dot{r}_{3}\right)
\end{aligned}
$$


where the sums denote Clebsch-Gordon series restricted to terms having the superskewsymmetry of the graded bracket by choosing

$$
\widehat{\Gamma}(S, R)=\{(v, \dot{v}) \in \Gamma(S, R) \mid v+\dot{v}=(s+\dot{s})+(r+\dot{r})-4(s+\dot{s})(r+\dot{r})-1 \bmod 2\} .
$$

Curvature components thus defined form curvature tensors $F(S, R)$ for all pairs $S, R \in \Lambda_{p}$.

Generalised self-duality [2, 3] corresponds to setting the following curvature representations to zero,

$$
F(v, \dot{s}+\dot{r})=0 \quad \text { for all } \quad v \in \gamma(s, r) \text { and }(s, \dot{s}),(r, \dot{r}) \in \Lambda_{p} .
$$

Similarly, generalised anti-self-duality corresponds to the imposition of the constraints

$$
F(s+v, \dot{v})=0 \quad \text { for all } \quad \dot{v} \in \gamma(\dot{s}, \dot{r}) \text { and }(s, \dot{s}),(r, \dot{r}) \in \Lambda_{p} .
$$

A third class of interesting constraints are the light-like integrable systems

$$
F(s+r, \dot{s}+\dot{r})=0 \quad \text { for all }(s, \dot{s}),(r, \dot{r}) \in \Lambda_{p} .
$$

All three classes are $S O(4, \mathbb{C})$-covariant equations. They are, moreover, amenable to generalised twistor-like transforms (for example, on the lines of the discussion in [6]). These systems therefore provide an infinitely large hierarchy of gauge- and Lorentz-covariant solvable systems. The corresponding linear (Lax) systems are given in [3].

For example, the simple super-Poincaré set, $\Lambda_{1}=\left\{\left(\frac{1}{2}, \frac{1}{2}\right),\left(\frac{1}{2}, 0\right),\left(0, \frac{1}{2}\right)\right\} \equiv\{V, S, \bar{S}\}$, has the set of a priori non-zero curvature tensors,

$$
\begin{aligned}
& \widehat{\Gamma}(V, V) \Leftrightarrow \quad\left\{F_{V V}(0,1), F_{V V}(1,0)\right\} \\
& \widehat{\Gamma}(V, S) \Leftrightarrow\left\{F_{V S}\left(1, \frac{1}{2}\right), F_{V S}\left(0, \frac{1}{2}\right)\right\} \\
& \widehat{\Gamma}(V, \bar{S}) \Leftrightarrow\left\{F_{V \bar{S}}\left(\frac{1}{2}, 1\right), F_{V \bar{S}}\left(\frac{1}{2}, 0\right)\right\} \\
& \widehat{\Gamma}(S, S) \Leftrightarrow\left\{F_{S S}(1,0)\right\} \\
& \widehat{\Gamma}(S, \bar{S}) \Leftrightarrow\left\{F_{S \bar{S}}\left(\frac{1}{2}, \frac{1}{2}\right)\right\} \\
& \widehat{\Gamma}(\bar{S}, \bar{S}) \Leftrightarrow\left\{F_{\bar{S} \bar{S}}(0,1)\right\}
\end{aligned}
$$

For this choice, the above three sets of zero curvature tensors correspond to the following:

\begin{tabular}{|c|c|c|}
\hline$F(v, \dot{s}+\dot{r})$ & $F(s+v, \dot{v})$ & $F(s+r, \dot{s}+\dot{r})$ \\
\hline$F_{V V}(0,1)$ & $F_{V V}(1,0)$ & \\
$F_{V S}\left(1, \frac{1}{2}\right), F_{V S}\left(0, \frac{1}{2}\right)$ & $F_{V S}\left(1, \frac{1}{2}\right)$ & $F_{V S}\left(1, \frac{1}{2}\right)$ \\
$F_{V \bar{S}}\left(\frac{1}{2}, 1\right)$ & $F_{V \bar{S}}\left(\frac{1}{2}, 1\right), F_{V \bar{S}}\left(\frac{1}{2}, 0\right)$ & $F_{V \bar{S}}\left(\frac{1}{2}, 1\right)$ \\
$F_{S S}(1,0)$ & $F_{S S}(1,0)$ & $F_{S S}(1,0)$ \\
$F_{S \bar{S}}\left(\frac{1}{2}, \frac{1}{2}\right)$ & $F_{S \bar{S}}\left(\frac{1}{2}, \frac{1}{2}\right)$ & $F_{S \bar{S}}\left(\frac{1}{2}, \frac{1}{2}\right)$ \\
$F_{\bar{S} \bar{S}}(0,1)$ & $F_{\bar{S} \bar{S}}(0,1)$ & $F_{\bar{S} \bar{S}}(0,1)$ \\
\hline
\end{tabular}

These correspond respectively to the supersymmetrisation of the standard four-dimensional self-duality condition, $F_{V V}(0,1)=0$, the supersymmetrisation of the anti-self-duality condition, $F_{V V}(1,0)=0$, and the 'conventional constraints' of $N=1$ supersymmetric gauge theory. Further examples are discussed in [3], including the celebrated $N=3$ constraints which are equivalent to the full equations of motion. 


\section{Concluding remarks}

Although we remain in the realm of supercommutative geometry, with $[\mathcal{V}, \mathcal{V}]_{\bullet}=0$, a generalisation to non-supercommutative geometry is clearly a further possibility, with the simplest superalgebra variant having $[.,]:. \mathcal{V} \times \mathcal{V} \rightarrow \mathcal{V}$ such that $\left[\mathcal{V}_{\alpha}, \mathcal{V}_{\beta}\right] \bullet \subset \mathcal{V}_{\alpha+\beta}$. Further generalisations, replacing this superalgebra structure, for instance, by $q$-deformed supercommutation relations, may also be considered along the lines of the present investigation.

We have considered an element of $\mathcal{A}, \mathcal{V}$ to be of bosonic type if its spin $(s+\dot{s})$ is an integer and of fermionic type if its spin is a genuine half-integer; and we have assumed the corresponding statistics. We note, however, that this assignment is a purely conventional one, motivated by the spin-statistics theorem. This can indeed be lifted, if required, to yield Lie algebra (rather than superalgebra) extensions of the Poincaré algebra containing integer and half-integer spin elements, all of even statistics. Such algebras maintain, nevertheless, their $\mathbb{Z}_{2}$-graded nature [7]. Such a variant of the supersymmetry algebra was recently shown to be the target space symmetry of the $N=2$ string [8] and the space of string physical states was shown to be elegantly and compactly describable in terms of a self-dual field on a hyperspace with a vectorial and an even-spinorial coordinate. On such hyperspaces with even spinorial coordinates, the generalised self-duality equations are the same as those given above; the only difference being that the a priori set of curvature components is determined from commutators, rather than supercommutators, between covariant derivatives.

\section{References}

[1] E.S. Fradkin and M.A. Vasiliev, Candidate for the role of higher spin symmetry, Ann.Phys. 177 (1987) 63; M.A. Vasiliev, Consistent equations for interacting massless fields of all spins in the first order in curvatures, Ann.Phys. 190 (1989) 59.

[2] C. Devchand and J. Nuyts, Self-duality in generalised Lorentz superspaces, hep-th/9612176, Phys. Lett. B404 (1997) 259.

[3] C. Devchand and J. Nuyts, Supersymmetric Lorentz-covariant hyperspaces and self-duality equations in dimensions greater than (4)4), hep-th/9704036, Nucl. Phys. B503 (1997) 627.

[4] C. Devchand and J. Nuyts, Lorentz covariant spin two superspaces, hep-th/9804052, Nucl. Phys. B (1998).

[5] D.A. Varshalovitch, A.N. Moskalev, V.K. Khersonskii, Quantum Theory of Angular Momentum, World Scientific Publishing Company, Singapore (1988).

[6] C. Devchand and V. Ogievetsky, Four-dimensional integrable theories, hep-th/9410147, in Lect. Notes in Physics 447, Springer-Verlag (1995).

[7] D.V. Alekseevsky and V. Cortés, Classification of N-(super)-extended Poincaré algebras and bilinear invariants of the spinor representation of Spin $(p, q)$, Commun. Math. Phys. 183 (1997) 477.

[8] C. Devchand and O. Lechtenfeld, Extended self-dual Yang-Mills from the $N=2$ string, hep-th/9712043, Nucl. Phys. B516 (1998) 255. 